\title{
Control del proceso de deshumidificación de muros con georradar. Un edificio patrimonial como caso de estudio.
}

\author{
S. Gea ${ }^{1}$, R. Quinteros ${ }^{2}$, L. Nallim ${ }^{2}$
}

${ }^{1}$ Facultad de Ingeniería, Consejo de Investigación, Universidad Nacional de Salta, Argentina

${ }^{2}$ Facultad de Ingeniería, Consejo de Investigación, Universidad Nacional de Salta, INIQUI-CONICET, Argentina.

\begin{abstract}
Información del artículo
DOI:

http://dx.doi.org/10.21041/ra.v4

$\underline{i 1.62}$

Artículo recibido el 29 de

septiembre de 2013, revisado

bajo las políticas de publicación

de la Revista ALCONPAT y

aceptado el 10 de enero de

2014. Cualquier discusión,

incluyendo la réplica de los autores, se publicará en el tercer número del año 2014 siempre y cuando la información se reciba antes del cierre del segundo número del año 2014.
\end{abstract}

\section{(C) 2014 ALCONPAT Internacional}

\section{Información Legal}

Revista ALCONPAT, Año 4, No. 1, Enero Abril 2014, es una publicación cuatrimestral de la Asociación Latinoamericana de Control de Calidad, Patología y Recuperación de la Construcción, Internacional, A.C., Av. Zamná No. 295 entre 61 y 63 Fraccionamiento Yucalpetén, Mérida, Yucatán, México, C.P. 97248, Tel.5219997385893.

97248, Tel.5219997385893,
alconpat.int@ gmail.com, Página Web: www.alconpat.org

Editor responsable: Dr. Pedro Castro Borges Reserva de derechos al uso exclusivo No.04 2013-011717330300-203, eISSN 2007-6835, ambos otorgados por el Instituto Nacional de Derecho de Autor. Responsable de la última Derecho de Autor. Responsable de la ultima actualización de este número, Unidad de Inforizabeth Sabido Maldonado, Av. Zamná No. 295 entre 61 y 63 Fraccionamiento Yucalpetén, Mérida Yucatán, México, C.P. 97248 , fecha de publicación: 30 de enero de 2014.

Las opiniones expresadas por los autores no necesariamente reflejan la postura del editor. Queda totalmente prohibida la reproducción total o parcial de los contenidos e imágenes de la publicación sin previa autorización de la ALCONPAT Internacional A.C.

\section{RESUMEN}

En edificios históricos son conocidos los efectos nocivos de la presencia de humedad en muros, que deteriora detalles de ornamentación, conjuntamente con los componentes de la mampostería que conforma su propia estructura. Es el caso de la Iglesia Nuestra Señora de la Viña (Salta, Argentina), edificio del s. XIX que presentaba manifestaciones patológicas características de las producidas por la presencia de humedad ascendente. Las tareas de recuperación de las superficies exteriores fueron realizadas una vez que el sistema instalado (por electrólisis) deshumidificó los muros. La elevada inversión realizada requiere de un control periódico de la eficacia del sistema. En este trabajo se presentan los fundamentos y los resultados obtenidos con el empleo de georradar como recurso no destructivo para controlar el sistema de deshumidifación, cuya principal ventaja es la de poder detectar en forma precoz a lo largo del tiempo alguna falla en el sistema, evitando en forma indefinida la aparición de nuevas manifestaciones patológicas por humedad ascendente.

Palabras clave: patología; mampostería; humedad; radar.

\section{ABSTRACT}

In historical buildings the harmful effects in masonry due to moisture are well known, as it affects ornamentation and the components of the masonry which is also the building's structure. That is the case of the church Nuestra Señora de la Viña (Salta, Argenti na), a XIX cent. Building which presented typical pathological symptoms caused by rising damp. An electro physical system was installed in order to dehumidificate the masonry and external surfaces were subsequently recovered. This high investment requires a periodical control of the system's efficiency. This article presents the foundations of and the results obtained by using an impulse radar as a nondestructive resource to control the dehumidification system. Its main advantage is the early detecting of a failure in the system and so avoiding new pathological symptoms caused by rising damp.

Keywords: pathology; masonry; moisture; radar.

Autor de contacto: Susana Gea (geas@unsa.edu.ar) 


\section{INTRODUCCIÓN}

La ciudad de Salta (Argentina) posee un centro histórico con edificios de valor patrimonial, entre los que se encuentra la iglesia Nuestra Señora de la Candelaria de la Viña, construida en el s. XIX. Con un marcado estilo italianizante, con techos y paredes interiores revestidas de frescos, su diseño y proporciones la convierten en uno de los edificios históricos más importantes de la ciudad, habiendo sido declarada Monumento Histórico Nacional en el año 1982 (Figura 1). Este edificio fue construido con muros de cal y canto, de alrededor de $80 \mathrm{~cm}$ de espesor, combinando ladrillo cerámico macizo, bloques de piedra canteada (areniscas cuarzosas) y mortero, como se aprecia en las Figuras 2(c) y 2(d).

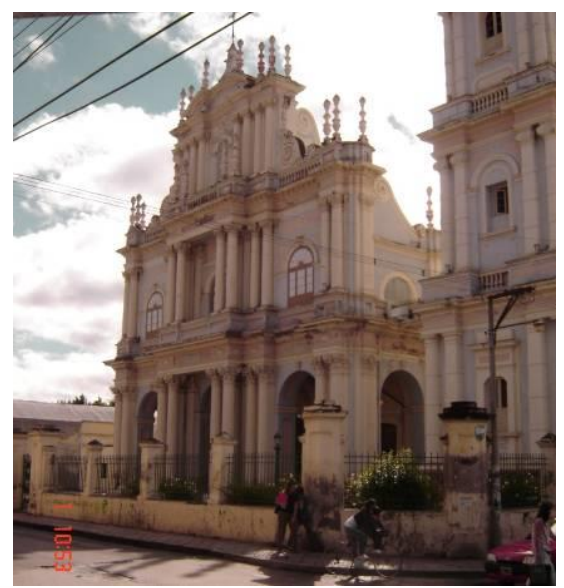

(a)

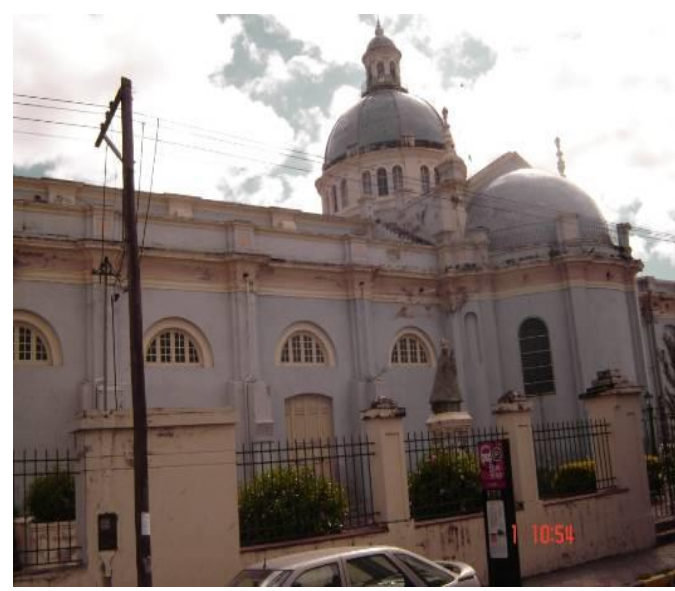

(b)

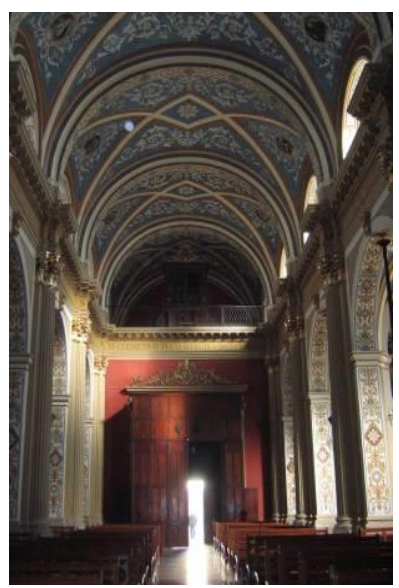

(c)

Figura 1. Iglesia Ntra. Sra. de la Viña: (a) Fachada principal;

(b) fachada lateral sur; (c) interior

Los revoques y morteros de asiento de cal y arena constituyeron un buen vehículo de transporte de la humedad ascendente desde el terreno, ya que los muros carecen de impermeabilización horizontal. Las sales solubles contenidas en el agua se depositaron en la superficie, la que luego al evaporarse y cristalizar dichas sales fueron deteriorando revoques y pinturas. Algunos muros presentaban daños por esta causa (Figura 2).

Con el fin de preservar el edificio, se instaló un equipo que emite una frecuencia modulada, sincronizada y mezclada que produce en la pared un circuito eléctrico de corriente separado, provocando una baja en la línea de potencial y forzando al agua contenida en los poros de la mampostería a volver al terreno e impidiendo que vuelva a ascender.

El proveedor del equipo garantiza que la humedad de los muros se mantendrá por debajo de $3.5 \%$ en peso y realiza controles periódicos del contenido de humedad de la mampostería, empleando el método de la resistencia. Para ello fueron dejados insertos pares de electrodos en distintos sectores del edificio, localizados $10-20 \mathrm{~cm}$ por encima del piso.

Como se mencionó anteriormente, la mampostería está constituida por materiales cuya porosidad, y por lo tanto la absorción de humedad, es muy diferente para cada uno de ellos (Tabla 1) y como se observa en la Figura 2, la piedra incluida en los muros puede alcanzar gran tamaño (hasta $60 \mathrm{~cm}$ de lado). Estas características de los materiales generan importante inhomogeneidad de los muros y por lo tanto, incertidumbres respecto de las mediciones que se describen en el párrafo anterior.

Más aún, en la fachada del atrio se observa en la actualidad algún deterioro en la pintura recientemente aplicada - como se verá más adelante - lo que muestra fallas en el sistema de deshumidificación. 


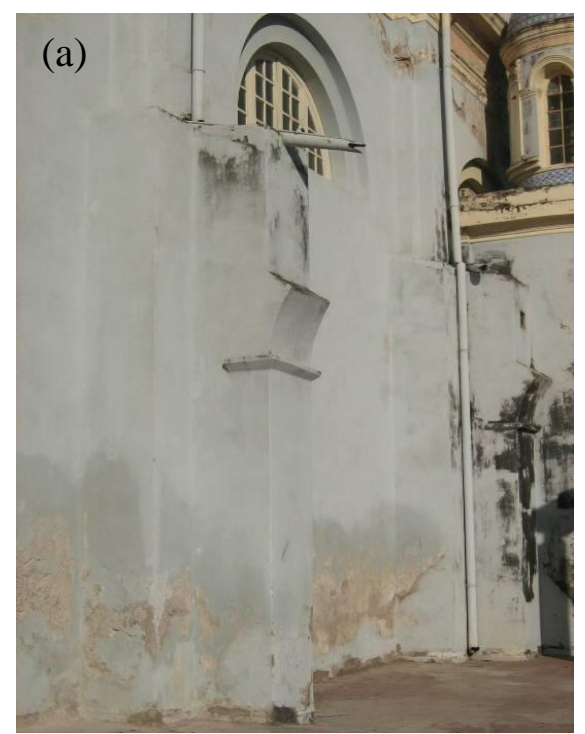

(c)

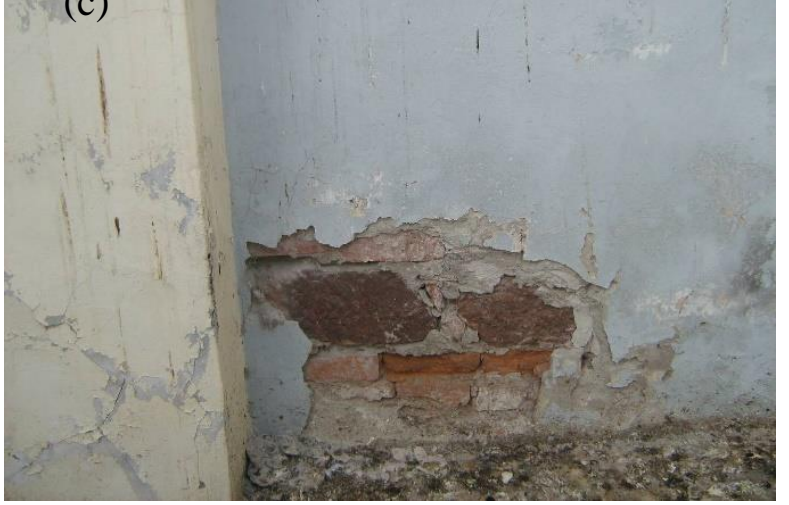

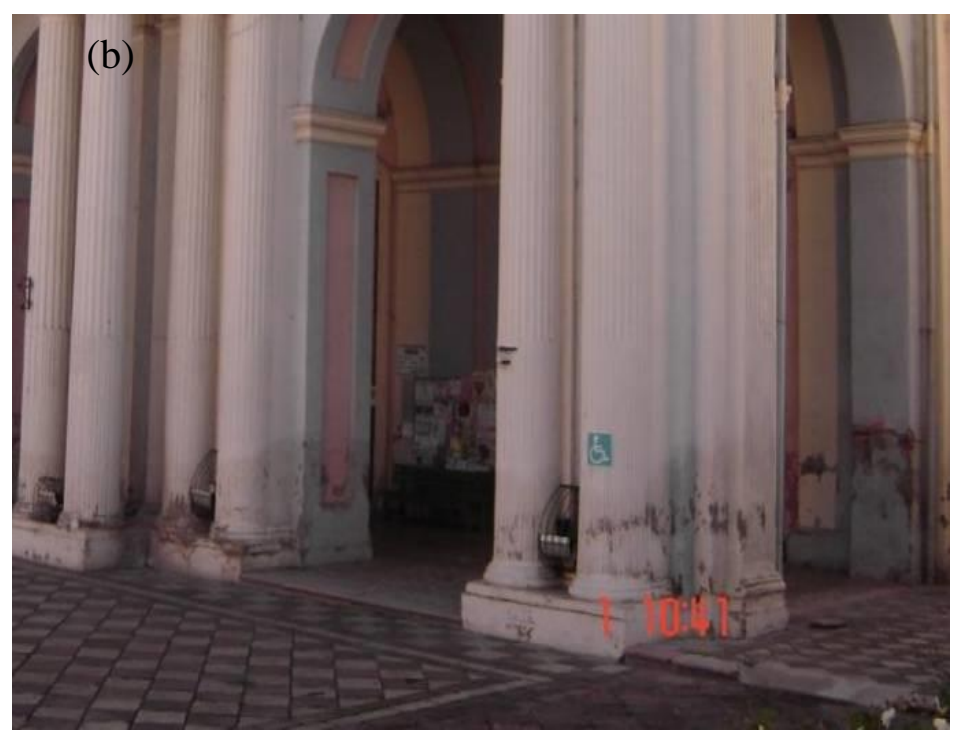

(d)

Figura 2. (a) y (b) Humedad en muros de fachada; (c) y (d) deterioro de revoques y mampuestos por el proceso de humedecimiento y secado.

Tabla 1. Absorción de humedad de los distintos materiales constituyentes de la mampostería

\begin{tabular}{|c|c|}
\hline Material & $\begin{array}{c}\text { \% de absorción de } \\
\text { agua en peso }\end{array}$ \\
\hline Piedra arenisca & $<2$ \\
\hline Mortero & $>15$ \\
\hline Ladrillo cerámico & $>20$ \\
\hline
\end{tabular}

\section{SISTEMA DE CONTROL DE DESHUMIDIFICACIÓN CON GEORRADAR}

\subsection{Fundamentos.}

Se presenta a continuación una metodología alternativa para determinar presencia de humedad por medio de georradar, o radar de penetración del terreno (GPR).

El GPR basa su funcionamiento en la propagación de cortos impulsos electromagnéticos, los que se transmiten a través del material del edificio empleando una antena dipolo. Los impulsos son reflejados en las interfaces entre los materiales con diferentes propiedades dieléctricas (por ejemplo, en la superficie y parte posterior de los muros, en los descascaramientos, en oquedades) (Binda, Lualdi, 
Saisi; 2007). Cuando se mueve la antena a lo largo de la superficie del objeto que se investiga, las imágenes del radar se producen en una escala de grises, que dan la intensidad de los ecos del radar como una función de la posición de la antena y el tiempo de paso de ondas. Midiendo el tiempo entre la emisión y el eco de la onda, y conociendo la velocidad de propagación en el medio, es posible conocer la profundidad del obstáculo en la pared. En los casos reales, la velocidad puede calcularse si se conoce el espesor del muro y con este valor se puede determinar el dieléctrico del material.

A tal fin, se recuerda que la relación entre la velocidad de propagación de una onda electromagnética en el vacío, la permeabilidad magnética en el vacío y la permitividad dieléctrica en el vacío está dada por la expresión (1), donde c es la velocidad de propagación de una onda electromagnética en el vacío.

$c=1 / \sqrt{\varepsilon_{0} \mu_{0}}=2,999 \times 10^{8} \mathrm{~m} / \mathrm{s}$,

Donde $\mu_{0}=4 \pi \times 10^{-7} \mathrm{H} / \mathrm{m}$ y $\varepsilon_{0}=8.854 \times 10^{-12} \mathrm{~F} / \mathrm{m}$.

Se expresa la velocidad de propagación de la onda en el medio como:

$c=1 / \sqrt{\varepsilon_{r}}$

Siendo $\varepsilon_{\mathrm{r}}$ es la constante dieléctrica del material.

La técnica sirve también para obtener información sobre la presencia de humedad en el interior de la mampostería. La humedad se revela por una disminución de la velocidad de las ondas y por un incremento de su atenuación. Esto está claramente demostrado (Binda y Saisi; 2009) por ensayos de laboratorio: la Figura 3 muestra el radargrama producido por una antena de $1 \mathrm{GHz}$ que se desplaza verticalmente hacia abajo $2 \mathrm{~m}$ en un muro de mampostería tradicional de ladrillos y en el que se observa que la humedad ascendente del suelo produce un gradual incremento del tiempo de reflexión. El dieléctrico, para el caso del agua a $20^{\circ} \mathrm{C}$, asciende a 81 mientras que para todo el resto de los materiales, el parámetro queda comprendido entre 1 y 81 . En el caso de estudio, se acepta un valor de $\varepsilon r$ comprendido entre 3 y 8 aproximadamente, ya que este depende directamente de la composición de los muros, tanto de las propiedades de los materiales componentes como de su participación volumétrica.

Si se conocen las dimensiones de lo que se está analizando o si es posible medirlas, entonces podemos conocer la velocidad promedio de propagación del medio. En nuestro caso de estudio, los espesores de los muros son conocidos, por lo que mediante la expresión (2) es posible calcular el valor promedio de $\varepsilon_{\mathrm{r}}$ o constante dieléctrica del material.

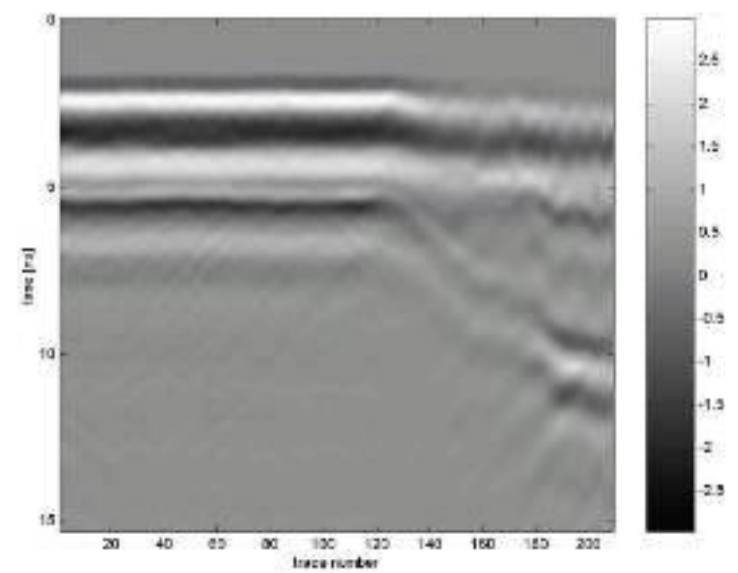

Figura 3. Datos de GPR de un perfil en una mampostería de ladrillos parcialmente húmeda. El efecto del contenido de humedad se observa en el lado derecho de la sección. (Binda y Saisi; 2009) 
Topp (1980) investigó de manera empírica la relación que existe en los suelos entre el valor del dieléctrico y el contenido volumétrico de agua en el medio, mediante la cual es posible conocer uno de esos parámetros en función del otro conocido, relación que queda determinada por las siguientes expresiones:

$$
\varepsilon_{r}=3.03+9.3 \theta+146.0 \theta^{2}-76.7 \theta^{3}
$$

con:

$\varepsilon_{r}=$ constante dieléctrica del material

$\theta=$ contenido volumétrico de agua

y su inversa:

$$
\theta=-5.310^{-2}+2.9210^{-2} \varepsilon_{r}-5.510^{-4} \varepsilon_{r}^{2}+4.310^{-2} \varepsilon_{r}^{3}
$$

La determinación de $\theta$ con la expresión (4) presenta errores de diversa magnitud según sea el tipo de matriz del terreno, pudiendo ser tenido en cuenta con la densidad volumétrica del mismo, según la expresión (5) (Pavlik et al., 2006)

$\theta=\frac{\varepsilon_{r}^{0.5}-0.819-0.168 \rho-0.159 \rho^{2}}{7.17+1.18 \rho}$

Estas expresiones pueden ser extendidas a otros geomateriales, como la mampostería. Sin embargo, si resulta necesario obtener información cuantitativa sobre el contenido de humedad, es preciso una calibración específica para cada material (Mollo y Greco, 2011). Esto, en edificios históricos no es posible realizar, ya que el mortero varía sus características de porosidad incluso dentro del mismo muro.

\subsection{Procedimiento experimental y procesamiento de datos}

Se empleó un georradar GSSI SIR-3000 con una antena de $900 \mathrm{MHz}$, que tiene un alcance de alrededor de $1 \mathrm{~m}$, y por lo tanto, adecuada para los muros de la iglesia que tienen en general 0,84m de espesor. Se incorporó un odómetro a la antena, haciendo innecesario controlar la velocidad de barrido. El procesamiento de los registros tomados en la obra se realizó con el software RADAN 6.5 (GSSI, 2006).

En primer lugar, fue necesario investigar sobre la constitución interna de los muros; es decir, determinar si estos fueron construidos en una o varias láminas verticales (en este último caso, con o sin relleno entre láminas). La Figura 4 muestra un radargrama post-procesado que permite visualizar la constitución interna de uno de los muros (Aldana, Ceballos, Gea; 2011), mostrando que se trata de muro macizo de una única lámina. Se pudo verificar con el georradar el espesor del revoque, de $3 \mathrm{~cm}$, que había sido determinado en los sectores en los que se encontraba dañado (Figuras 2(c) y 2(d)). 


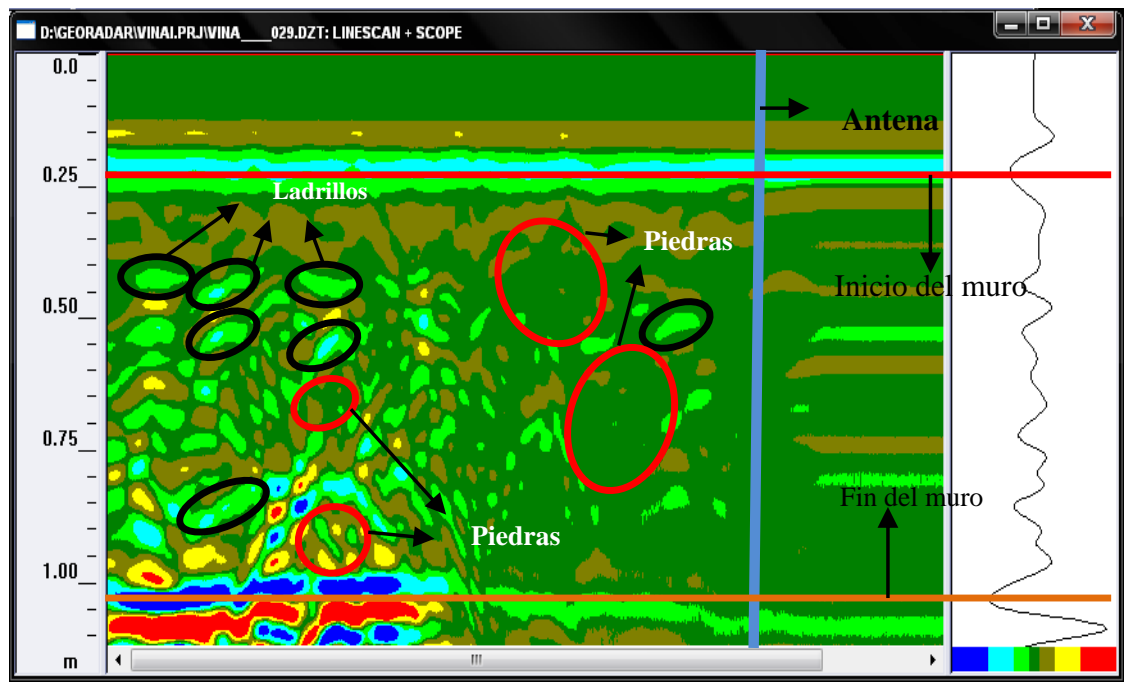

Figura 4. Radargrama procesado que permite visualizar el interior del muro

Con el fin de determinar el contenido de humedad del edificio se tomaron registros en dirección vertical, desde el nivel de piso y hasta $2 \mathrm{~m}$ de altura (Figura 5a). Las mediciones fueron realizadas tanto sobre la cara exterior como la interior de los muros, siguiendo en cada sector la misma línea vertical. Esta redundancia en las mediciones permite mejorar la interpretación y detectar errores en los registros. En la Figura 5(b) se indican los sectores investigados.

Para una correcta interpretación de los resultados obtenidos tras la medición, se utilizan los filtros del software destinado para el procesamiento de los registros, con lo que se busca disminuir las interferencias externas y así limpiar la señal registrada, es decir mejorar la visual del registro para una mejor interpretación (Santos Assunção y Pérez-Gracia, 2011).

\subsection{Resultados obtenidos}

A continuación se muestran resultados obtenidos en dos sectores de medición, a modo de ejemplo, que se indican en la Figura 5(b) como [1] y [2]. Ambos corresponden a la fachada del atrio. La Figura 5(a) muestra el sector [1], el cual tiene las mismas características arquitectónicas que el sector [2]. En la Figura 5(c) se puede observar un detalle de este último, con daños en la pintura.

2.3.1 Fachada atrio (Sector 1): En la Figura 6(a) se muestra el registro sin procesar obtenido del sector 1. Las Figuras 6(b) y 6(d) muestran el resultado obtenido luego de la limpieza de la señal registrada mediante de la aplicación de algunos filtros del software tales como el de corrección de posición inicial o retiro, o el filtro de "remoción de fondo" (background removal), que se utiliza para remover ruidos que son constantes en los registros y se deben a interferencias de la propia antena y reverberaciones de las ondas en el medio. En la Figura 6(b) se destacan algunos puntos notorios cuya velocidad de propagación es fácil de distinguir debido a la reflexión de las ondas en el medio. 

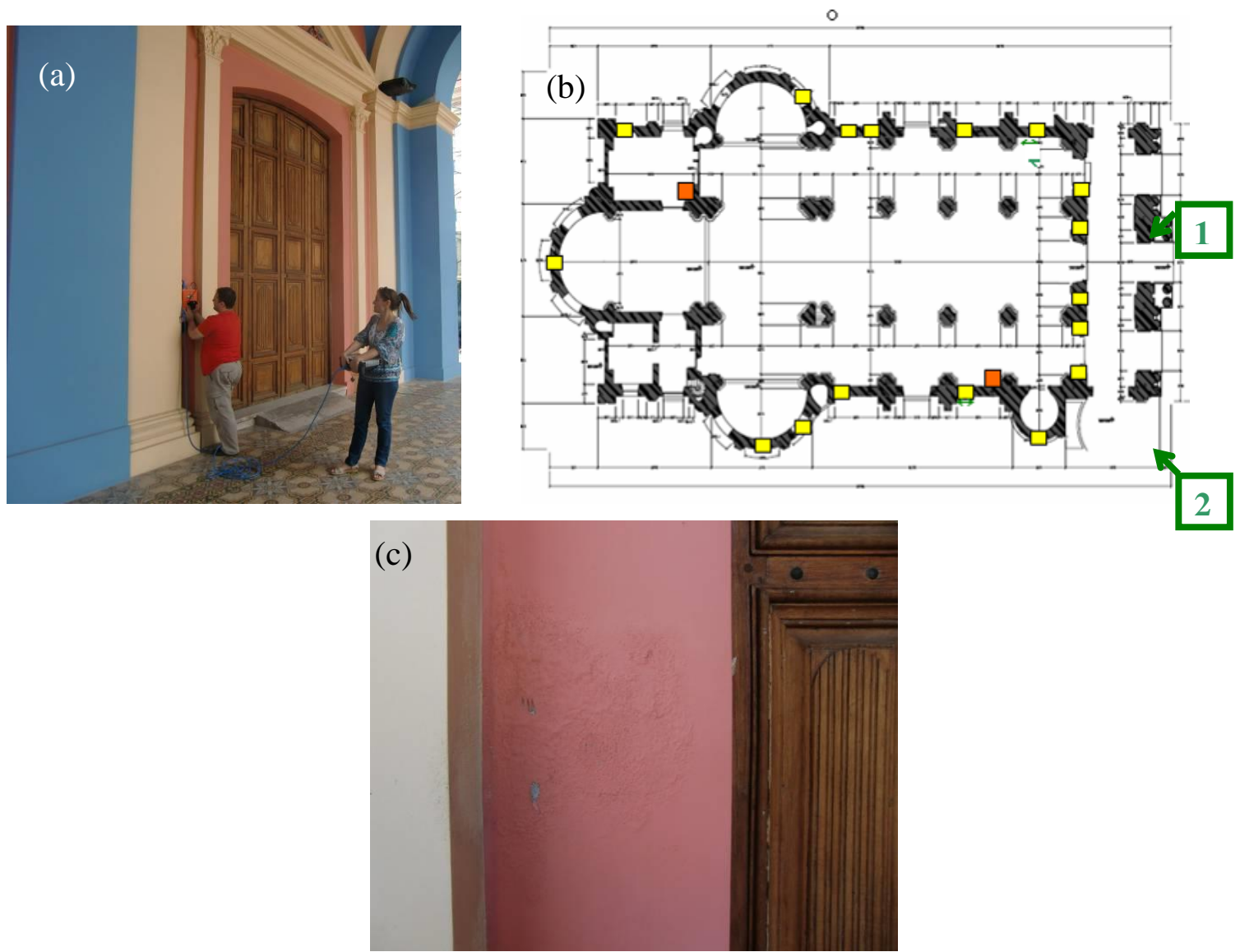

Figura 5. (a) Barrido con antena de 900MHz en dirección vertical en el sector [1] de la fachada del atrio; (b) sectores analizados en color amarillo y localización del equipo de deshumidificación en color naranja; (c) detalle de daño en pintura en sector [2].

2.3.2 Fachada atrio (Sector 2): En la Figura 7(d) se puede suponer de manera aproximada la distribución de la humedad en el muro a lo largo de su altura en analogía con la distribución de los valores de las velocidades de propagación. Esto es, mientras más bajo es el valor de la velocidad (zonas más oscuras) más alto es el contenido de humedad o presencia de agua en el medio; mientras que si la velocidad crece (zonas más clara), la humedad disminuye. 

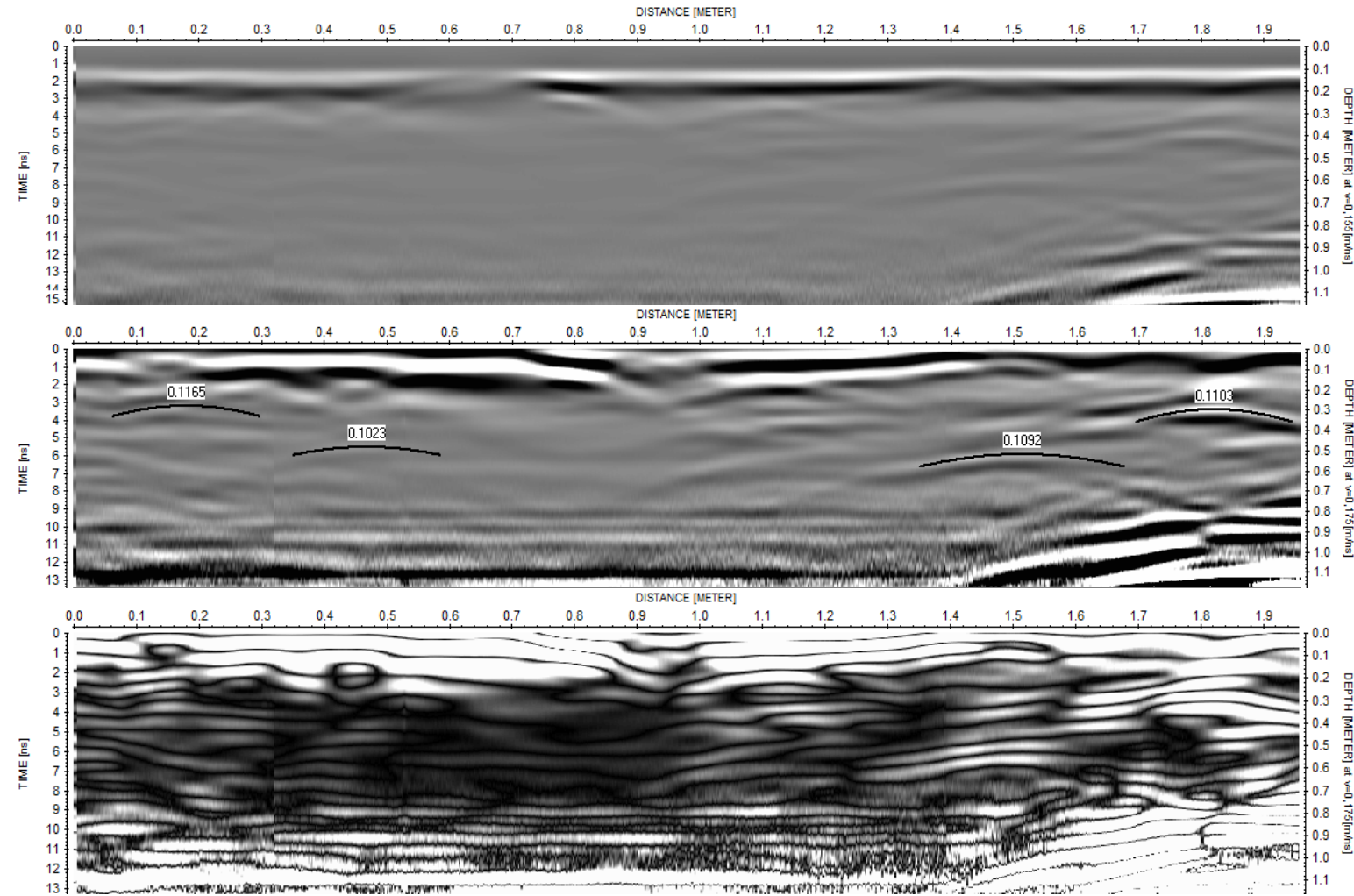

Figura 6. Registros en el sector 1: (a) sin procesar, b) procesado indicando velocidades de propagación puntuales y (c) procesado con incremento de contrastes
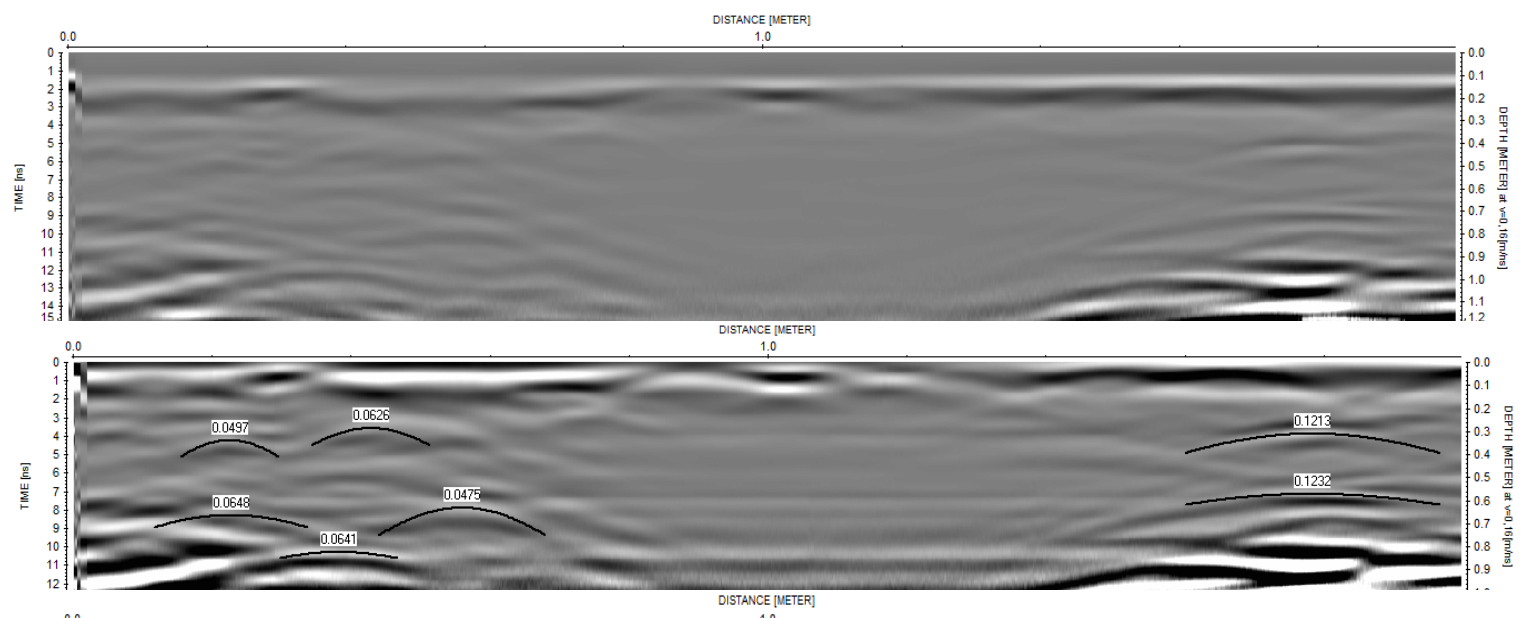

(b)

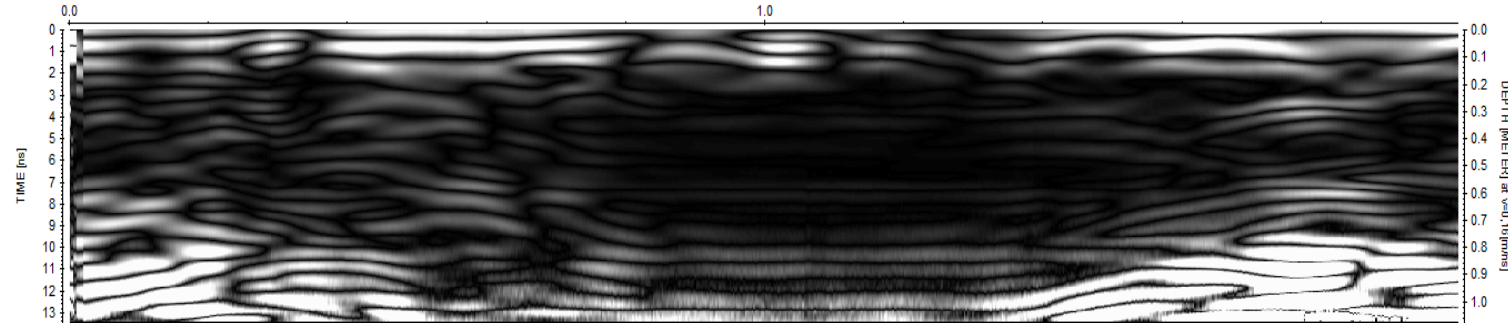

(c)

Figura 7. Registros en el sector 2: (a) sin procesar, (b) procesado indicando velocidades de propagación puntuales, (c) procesado con incremento de contrastes 
Revista ALCONPAT, Volumen 4, Número 1, Enero - Abril 2014, Páginas 74 - 83

\section{DISCUSIÓN}

Para determinar el contenido porcentual de humedad, se tuvo en cuenta la inhomogeneidad de los muros. El GPR permite identificar los bloques de piedra en sectores de los muros de cuyo estudio se prescinde, ya que esta tiene muy baja absorción (Tabla 1). Los valores de velocidad, dieléctrico y contenido de humedad fueron determinados solamente en los sectores de mampostería de ladrillo y mortero, para lo cual se tomó la densidad volumétrica $\rho=1.6 \mathrm{~g} / \mathrm{cm}^{3}$ (Gea et al., 2010). Las Tablas 2 y 3 resumen los valores obtenidos empleando las expresiones (2) y (5).

Asumiendo, como se indicó anteriormente, que la matriz del material que se analiza tiene una influencia directa en la determinación de humedad por medio del dieléctrico (Malicki, Plagge, Roth, 1996) los valores de $\theta$ obtenidos no se interpretan como valores absolutos, sino relativos: el sector 1 es un sector al que podemos calificar como seco, ya que se encuentra protegido de la intemperie y por lo tanto la única fuente posible de humedad es la del terreno; si la hubiera, esta sería máxima en las cercanías del piso, disminuyendo en altura hasta tomar un valor constante, correspondiente a la humedad natural del muro. Se observa en la Figura 6 y en la Tabla 2 que los valores de $\theta$ son prácticamente constantes en los casi $2 \mathrm{~m}$ de altura analizados, con un valor promedio $\theta_{1}=0.14$.

Tabla 2. Resultados obtenidos de las mediciones en el Sector 1

\begin{tabular}{|c|c|c|c|}
\hline $\begin{array}{c}\text { Cota desde el } \\
\text { nivel de piso } \\
{[\mathrm{m}]}\end{array}$ & $\begin{array}{c}\text { Velocidad } \\
{[\mathrm{m} / \mathrm{ns}]}\end{array}$ & $\varepsilon_{\mathrm{r}}$ & $\theta$ \\
\hline 0,18 & 0,1165 & 6,62 & 0,12 \\
\hline 0,46 & 0,1023 & 8,59 & 0,16 \\
\hline 1,50 & 0,1092 & 7,54 & 0,14 \\
\hline 1,82 & 0,1103 & 7,39 & 0,14 \\
\hline \multicolumn{3}{|c|}{ Promedio $\theta_{1}$} & 0.14 \\
\hline
\end{tabular}

Tabla 3. Resultados obtenidos de las mediciones en el Sector 2

\begin{tabular}{|c|c|c|c|}
\hline $\begin{array}{c}\text { Cota desde el } \\
\text { nivel de piso } \\
{[\mathrm{m}]}\end{array}$ & $\begin{array}{c}\text { Velocidad } \\
{[\mathrm{m} / \mathrm{ns}]}\end{array}$ & $\varepsilon_{\mathrm{r}}$ & $\theta$ \\
\hline 0,20 & 0,0497 & 36,39 & 0,50 \\
\hline 0,22 & 0,0648 & 21,40 & 0,35 \\
\hline 0,38 & 0,0641 & 21,87 & 0,35 \\
\hline 0,42 & 0,0626 & 22,94 & 0,36 \\
\hline 0,56 & 0,0475 & 39,83 & 0,53 \\
\hline 1,80 & 0,1213 & 6,11 & 0,11 \\
\hline \multicolumn{3}{|c|}{ Promedio $\theta_{2}$} & 0.37 \\
\hline
\end{tabular}

Tomamos este valor como referencia para analizar los resultados del resto de los sectores analizados. Como ejemplo de interés se presenta en este trabajo el sector 2, que adquiere el mínimo valor de $\theta$ recién a $1.80 \mathrm{~m}$ de altura y se observa (Tabla 3 ) que este crece hasta cuadruplicarse a $20 \mathrm{~cm}$ del piso. Esta clara manifestación de presencia de humedad ascendente confirma las causas del daño en la pintura y, por lo tanto, de fallas en el sistema de deshumidificación. 
Revista ALCONPAT, Volumen 4, Número 1, Enero - Abril 2014, Páginas 74 - 83

\section{CONCLUSIONES}

Se presenta un método rápido para determinar el contenido volumétrico de humedad por medio de georradar.

Tiene una gran ventaja frente a los métodos tradicionales de medición en puntos fijos (como por ejemplo el de la resistividad) en edificios antiguos construidos con mampostería de gran inhomogeneidad y absorción variable de los materiales componentes, ya que primeramente se identifica el material cuya humedad se está analizando y así se interpreta correctamente el resultado de la medición.

El contenido de humedad puede ser mapeado en la altura del muro y así identificar la fuente de la presencia de agua. Si bien no se puede determinar el contenido gravimétrico de humedad, la determinación de $\theta$ constante puede ser un criterio de aceptación del proceso de deshumidificación. Por último, se advierte que un estudio con GPR en forma periódica en el edificio permitirá la detección temprana de presencia de humedad, antes de que aparezcan daños en los revestimientos.

\section{REFERENCIAS}

Aldana G., Ceballos M. A. \& Gea S. (2011), “Experiencias con GPR en los procesos de evaluación de estructuras”, actas del $7^{\circ}$ Congreso Internacional sobre Patología y Rehabilitación de Estructuras, Fortaleza (Brasil), 2011.

Binda, L., Lualdi, M. \& Saisi, A., (2007) “Non-destructive testing techniques applied for diagnostic investigation: Syracuse Cathedral in Sicily, Italy”, International Journal of Architectural Heritage, 1: 380-402.

Binda, L. \& Saisi, A., (2009) "Application of NDTs to the diagnosis of Historic Structures". NDTCE'09, Non-Destructive Testing in Civil Engineering. Nantes, France, June 30th - July 3rd, 2009.

Gea, S., Toledo, M., Milia, S., Carrió, A. (2010), "Evaluación de la vulnerabilidad sísmica de la iglesia Nuestra Señora de la Candelaria de la Viña”. Actas de las XXXIV Jornadas Sudamericanas de Ingeniería Estructural. San Juan, Argentina, 27 al 30 de septiembre y 1 de octubre de 2010.

GSSI, Geophysical Survey Systems, Inc., 2006

Malicki, M. A., Plagge, R. \& Roth, C. H. (1996). "Improving the calibration of dielectric TDR soil moisture determination taking into account the solid soil”. European Journal of Soil Science, September 1996, 47,357-366

Mollo, L. \& Greco, R. (2011). "Moisture Measurements in Masonry Materials by Time Domain Reflectometry". J. Mater. Civ. Eng. 2011.23:441-444.

Pavlík, Z., Jiřičková, M., Černý, R., Sobczuk, H., and Suchorab, Z. (2006), "Determination of moisture diffusivity using the time domain reflectometry (TDR) method". Journal of Building Physics 2006 30: 59.

Santos Assunção, S., Perez-Gracia, V. (2011) "Mejoras en la visualización de señales de radar de subsuelo mediante el análisis frecuencial”. $12^{\circ}$ Congreso Nacional de Ensayos no Destructivos. Valencia, España.

Topp, G. C. (1980) "Electromagnetic determination of soil water content: measurements in coaxial transmission lines”. Water Resources Research, Vol. 16, No. 3, pp 574-582, June 1980 